\title{
Le lait à Moundou, témoin de l'intégration marchande des systèmes pastoraux au sud du Tchad
}

\author{
G. Duteurtre ${ }^{1}$ A. Atteyeh ${ }^{2}$
}

Mots-clés

Produit laitier - Pastoralisme Commercialisation Moundou - Tchad.

\section{Résumé}

Depuis 1980, l'importance de l'élevage dans la zone soudanienne du Tchad n'a cessé de croître à cause, notamment, de la descente de troupeaux transhumants du centre du pays vers le sud. L'approvisionnement des villes et des villages de la zone en produits laitiers représente une activité importante pour ces élevages. C'est sur cette base que se sont stabilisées des populations d'éleveurs pastoraux autour de la ville de Moundou. Ces élevages peuvent-ils répondre de manière durable à l'augmentation rapide de la demande urbaine ? Ces systèmes d'élevage se modifient-ils sous l'effet de l'intégration au marché ? Des enquêtes auprès de producteurs laitiers périurbains, de transformateurs laitiers, de commerçants et de consommateurs ont permis de mieux comprendre l'organisation de la filière. Les résultats ont souligné le dynamisme du commerce des produits laitiers traditionnels et l'importance des flux de produits entre les campements périurbains Peuls et les marchés de la ville (plus de 5000 litres par jour). Ces systèmes d'élevage ont d'ailleurs révélé des capacités d'adaptation au nouveau contexte marchand, tout en restant attachés à leur mobilité, même sur de faibles distances. D'autre part, la filière est apparue fortement dynamisée par le secteur de la transformation laitière urbaine (fromagers et bars laitiers). Enfin, la concurrence des importations est assez faible en raison de l'enclavement de la zone et de la préférence des consommateurs pour les produits traditionnels. Cette présence de l'élevage pastoral n'est cependant pas exempte de problèmes, comme celui concernant l'occupation de l'espace. Les résultats de cette étude soulignent les besoins de renforcer les initiatives de concertation entre éleveurs et agriculteurs, de favoriser une organisation plus efficace du marché du tourteau de coton et le développement des petits ateliers de transformation.

\section{INTRO DUCTION}

Le thème de l'insertion des systèmes pastoraux dans l'économie marchande en Afrique subsaharienne fait l'objet d'une littérature relativement abondante. Du Sahel à l'Afrique de l'Est, l'importance du commerce des produits laitiers dans l'économie pastorale est en général reconnue $(4,13)$. La part de ces systèmes dans la production laitière totale africaine est d'ailleurs estimée à 70 p. 100 (5).

Néanmoins, cette intégration au marché ne se fait pas toujours sans problèmes et les auteurs ne s'accordent pas tous entre eux sur les perspectives offertes par les systèmes pastoraux pour répondre au

1. Cirad-emvt, Prasac, Laboratoire de Farcha, BP 433, N'Djamena, Tchad Tél : +235 523007 ; fax : +235528302; e-mail : duteurtre@ cirad.fr

2. S/C Aden Darar Djibeh, BP 7310, République de Djibouti formidable défi de la croissance urbaine. Les élevages laitiers pastoraux peuvent-il contribuer de manière significative à l'approvisionnement des villes africaines ?

En Ethiopie, où la population humaine augmente nettement plus fortement que la population bovine, les élevages pastoraux seront à moyen terme incapables de produire des surplus suffisants pour participer de manière significative à l'approvisionnement des villes (4). De plus, Nell (10) estime que le potentiel de développement laitier des systèmes d'élevage pastoraux africains est insignifiant. D'autres auteurs soulignent au contraire que l'amélioration de l'alimentation des troupeaux en zone périurbaine pourrait permettre aux élevages pastoraux de répondre de manière compétitive à la demande urbaine, comme dans les villes du Nord Cameroun (11), de Mauritanie, de Djibouti ou du Sud marocain (6). La sédentarisation d'une partie de la famille ou d'une partie du troupeau laitier peut alors être une réponse adaptée à la demande du marché. D'autres études ont souligné les perspectives offertes par la collecte du lait en zone pastorale (14). 
Si l'on excepte les travaux de Le Masson (7) en République centrafricaine, peu d'études ont été réalisées sur le secteur laitier en zone soudanienne d'Afrique centrale. Pourtant, depuis 1980, le déplacement des éleveurs vers le sud, l'augmentation des surfaces cultivées et la forte croissance urbaine ont bouleversé le contexte dans lequel s'opère le commerce des produits laitiers (1). Plus récemment, la dévaluation du franc de la Communauté financière africaine (FCFA) en janvier 1994 a eu pour effet de renforcer, au moins pendant un temps, la compétitivité des productions locales.

Afin de mieux comprendre le rôle des systèmes pastoraux dans le commerce du lait en Afrique centrale, une étude a été menée sur la filière d'approvisionnement en produits laitiers de la ville de Moundou (sud du Tchad). Cette étude a fait l'objet d'un rapport de stage (2) dont les principaux éléments sont repris dans cet article. Après un rapide exposé de la méthode d'étude utilisée, l'article présente quelques résultats illustrant les évolutions des systèmes de production, de la commercialisation et de la demande. Une dernière partie discute des perspectives de l'étude en soulignant les défis de l'intégration au marché des systèmes pastoraux dans la zone des savanes d'Afrique centrale.

\section{MATERIEL ET METHODES}

L'étude des circuits d'approvisionnement en produits laitiers des villes africaines a été très largement abordée dans la littérature francophone grâce au concept de filière $(3,5,8)$. La filière est définie comme « la succession d'activités étroitement imbriquées les unes par rapport aux autres, liées verticalement à un même produit ou à des produits voisins et dont l'objectif principal, dans les filières agroalimentaires, semble être de répondre aux besoins de la demande $\gg(9)$.

L'approche filière prend en compte les itinéraires techniques (fonctions de production, de commercialisation, de transformation et de consommation), les stratégies et les relations des acteurs de la filière. Cette approche est particulièrement adaptée pour aborder dans leur diversité les systèmes d'approvisionnement de type traditionnel, artisanal ou moderne (5). Cinq niveaux d'investigation sont privilégiés :

- la décomposition des coûts et des marges dans la filière ;

- les diverses technologies utilisées (traditionnelles, artisanales, industrielles) ;

- les espaces de référence géographiques (local, national, régional international) ;

- les modes de coordination entre clients et fournisseurs (prix de marchés, contrats, hiérarchies, monopoles, etc.) ;

- les logiques des acteurs (comportements sécuritaires, spéculatifs, etc.).

La présente étude s'est basée sur des entretiens directs, conduits aux mois de mai et de juin 1999, auprès des acteurs de la filière.

1. Enquête auprès des producteurs : un questionnaire fermé a été soumis à un échantillon de 43 producteurs laitiers localisés autour de Moundou. Leur choix a été fait au hasard, suite à des reconnaissances des environs proches et éloignées de la ville à l'intérieur du rayon de collecte.

2. Enquêtes sur les marchés : les produits vendus sur les marchés, dans les épiceries, dans les bars et par les vendeurs ambulants ont été identifiés. Afin d'évaluer les quantités commercialisées, des comptages ont été réalisés sur les marchés et sur les principales voies d'approvisionnement de la ville pendant une journée.
Les prix de vente au détail des produits traditionnels pratiqués au moment de l'enquête ont été listés. Les prix des autres produits, qui sont fixés à l'avance, ont été relevés dans les points de vente. Les variations saisonnières ont été estimées " au dire d'acteurs ", c'est-à-dire sur la base de discussions avec les commerçants.

3. Enquêtes auprès des commerçants : cinq bars laitiers, deux fromageries et cinq importateurs ont été soumis à un questionnaire fermé et à un entretien ouvert.

4. Enquêtes auprès de consommateurs : dix-sept ménagères ont répondu à des questions selon un guide d'entretien semi-ouvert.

5. Pour compléter ces données d'enquêtes, des entretiens ouverts ont été conduits auprès des autorités publiques, de chercheurs et d'organisations non-gouvernementales.

\section{- RESU LTATS}

Avec 100000 habitants et une économie plutôt dynamique, Moundou fait figure de capitale régionale dans la zone soudanienne du Tchad. La croissance démographique y est relativement élevée et les activités agricoles périphériques se développent assez rapidement. La population autochtone est en majorité N'Gambaye et comprend aussi une minorité significative d'habitants originaires du Nord. La ville est bordée au sud par le Logone qui sépare les préfectures du Logone occidental et du Logone oriental.

La ville est approvisionnée en lait local à partir d'un bassin laitier s'étirant le long du fleuve sur une vingtaine de kilomètres à la périphérie sud de la ville (figures 1 et 2). Seul le lait de vache fait l'objet d'un commerce significatif. Cette ceinture laitière est caractérisée par une remarquable unicité ethnique des éleveurs. Ce sont des éleveurs Peuls du centre (Fulata baguirmi) et de l'ouest (Fulata ouda et Fulata haoussa), installés avant 1984. Certains notables ou commerçants moundoulais possèdent aussi des troupeaux importants qui sont laissés en gardiennage à des Peuls. En échange, le bouvier perçoit un salaire et dispose de la production laitière du troupeau. Ces animaux participent aussi à l'approvisionnement laitier de Moundou.

A côté de cet élevage pastoral, dont les caractéristiques sont décrites ci-après, certains agriculteurs N'Gambaye pratiquent un petit élevage bovin sédentaire. Ces animaux sont destinés à la traction attelée et ne participent pas à l'approvisionnement laitier de Moundou. Ce petit élevage ne fait donc pas l'objet d'une discussion dans le présent article, même s'il n'est pas exclu que les agriculteurs de la zone puissent à l'avenir jouer un rôle dans le commerce du lait.

\section{Les Peuls périurbains}

Les résultats des enquêtes menées auprès de 43 tentes d'éleveurs Peuls permettent de mieux connaître les techniques d'élevage et les stratégies pastorales. Sur l'ensemble de l'échantillon, 60 p. 100 sont uniquement des éleveurs, alors que 40 p. 100 pratiquent aussi l'agriculture de manière saisonnière. Tous les éleveurs pratiquent la transhumance sur de courtes distances et sont localisés en périphérie de la ville au moins une partie de l'année. Plusieurs raisons ont été évoquées pour expliquer le choix des éleveurs de s'implanter en zone périurbaine : l'insécurité qui règne dans les campagnes isolées, l'attrait du mode de vie urbain et la proximité des débouchés pour les produits laitiers.

Tous les éleveurs enquêtés, à l'exception d'un seul, établissent leurs campements de saison des pluies sur la rive méridionale $\mathrm{du}$ fleuve, dans le Logone oriental. Les familles rejoignent ces 


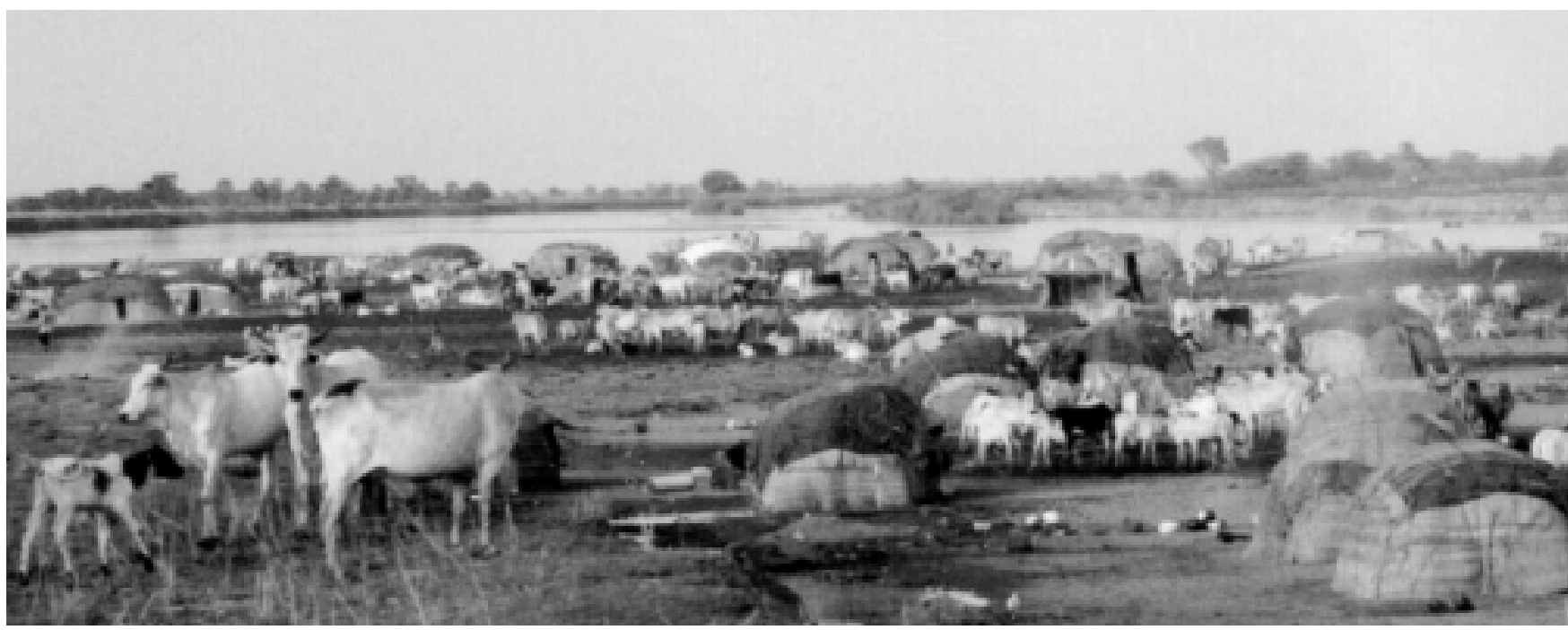

Figure 1 : campement Peul en bordure du Logone.

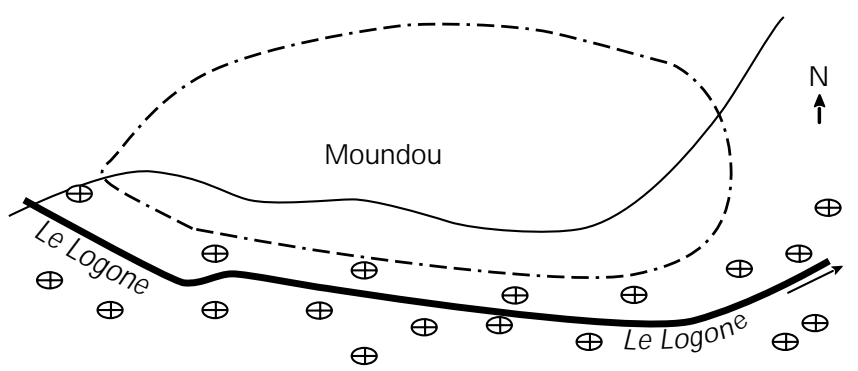

$\begin{array}{ll}\oplus- & \text { Limites de la zone urbaine } \\ \text { Localisation schématique des campements Peuls } \\ \text { Axe bitumé }\end{array}$

Figure 2 : localisation des campements d'éleveurs autour de Moundou (carte schématisée).

emplacements pendant les mois de juin (37 p. 100 de l'échantillon) ou de juillet (60 p. 100). C'est en cette saison que les troupeaux laitiers sont les plus éloignés des centres de consommation. Certains sont même conduits à dépasser le rayon de collecte qui s'étend sur vingt kilomètres et représente huit heures de marche aller-retour. La quasi-totalité de l'espace périurbain est mise en culture et beaucoup d'éleveurs conservent uniquement à proximité de la ville les vaches laitières pendant que le reste du troupeau est confié à des bouviers salariés ou à des membres de la famille. De juin à janvier, les pâturages et les résidus de culture assurent une alimentation jugée suffisante par les éleveurs.

Après la saison des pluies, les campements rejoignent leurs sites d'attache sur la rive gauche du Logone, en bordure proche de la ville. Ces déplacements ont lieu en octobre (42 p. 100 des éleveurs enquêtés), en novembre (44 p. 100) ou plus tard. Les troupeaux bénéficient alors de ressources alimentaires plus réduites. Pour maintenir une bonne production laitière de mars à mai, les éleveurs ont recours à des compléments. Même si les éleveurs reconnaissent avoir des difficultés pour s'approvisionner en tourteau de coton, environ 80 p. 100 affirment en distribuer aux vaches en lactation durant au moins une période de l'année.

D'autre part, l'accès au pâturage s'inscrit dans les relations difficiles qui existent entre les éleveurs et les agriculteurs sur les mêmes terroirs. Selon les éleveurs, les surfaces de pâturages diminuent sous l'effet des feux de brousse et surtout à cause de l'aug- mentation des surfaces emblavées. D'après eux, l'augmentation de la pression agricole serait due à la croissance démographique, aux nouveaux moyens de labour qu'offre la traction animale, à la mise en culture des couloirs de transhumance et des autres surfaces pâturées afin de bénéficier de la fumure, et à la priorité donnée par les chefs de cantons aux agriculteurs non-Peuls pour l'attribution des terres.

\section{Valorisation du lait chez les Peuls}

La traite est réalisée indifféremment par les époux (53 p. 100 des éleveurs visités), par les épouses (26 p. 100) ou par les enfants (21 p. 100). En revanche, dans tous les foyers visités, c'est la femme qui se charge de l'utilisation du lait : transformation, commercialisation et consommation familiale. La plupart des femmes font bouillir le lait juste après la traite.

Lorsqu'il n'est ni consommé ni vendu à l'état frais, le lait est laissé à fermenter pendant plusieurs heures, jusqu'à ce qu'il devienne acide. Le lait ainsi fermenté présente l'avantage de se conserver plusieurs jours à température ambiante et d'être plus facilement baratté.

Plusieurs techniques sont utilisées pour la fabrication du beurre. Chez les Fulata baguirmi, le lait fermenté est agité dans une grande calebasse fermée jusqu'à formation des granules de beurre. Chez les Fulata haoussa enquêtés, la technique utilisée est assurément plus performante. C'est la crème qui est barattée, non pas le lait fermenté. La crème recueillie en surface est versée dans une calebasse longue, de petite taille. Parfois, des cailloux peuvent être ajoutés dans la calebasse pour améliorer l'efficacité du barattage. Le barattage consiste alors à agiter la calebasse d'une main pendant que l'autre est laissée libre, le geste peut d'ailleurs être fait en position debout. Cette technique est donc beaucoup moins contraignante.

Lorsqu'elles ont réuni une quantité suffisante de lait, de lait fermenté ou de beurre, les femmes Peules se rendent à pied en ville pour écouler leurs produits (figure 3).

\section{Un large éventail de produits}

Les enquêtes sur les marchés montrent qu'un grand nombre de produits laitiers sont commercialisés à Moundou. Il s'agit, en 


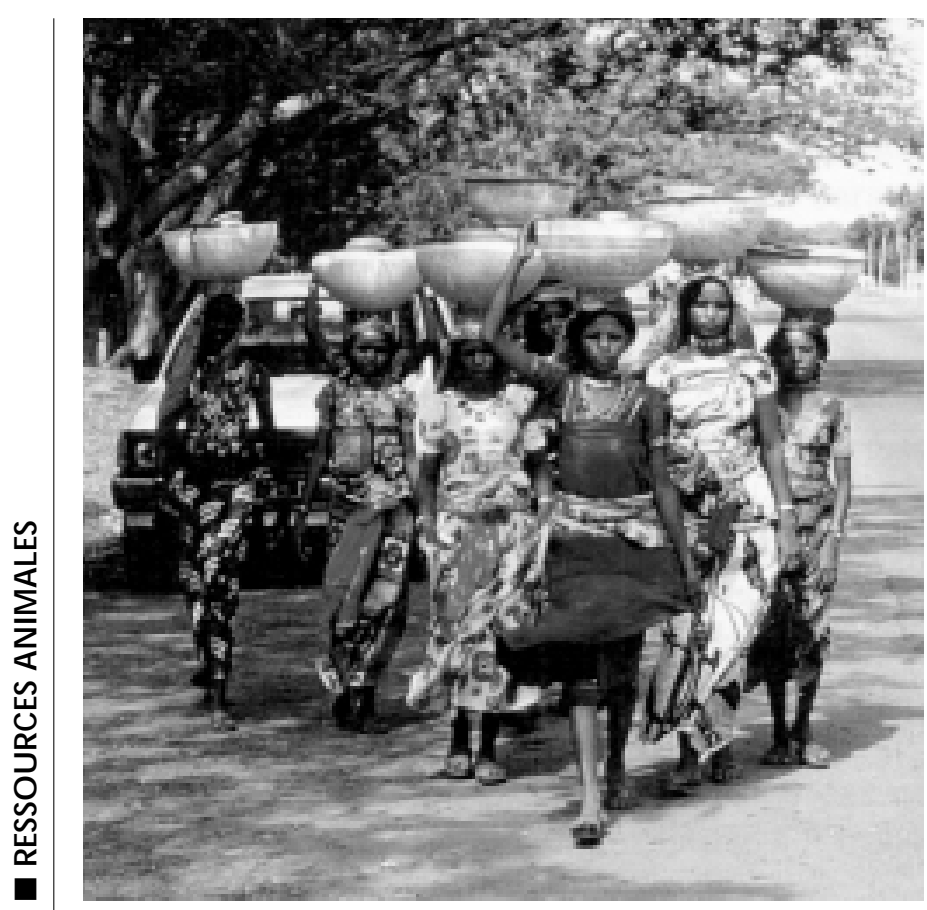

Figure 3 : livraison du lait à M oundou.

premier lieu, des produits traditionnels vendus par les femmes Peules : lait frais, lait caillé, beurre solide et beurre clarifié. On trouve aussi du lait fermenté sucré fabriqué et vendu par les bars laitiers. Certaines boutiques commercialisent des fromages frais de confection locale. Enfin, quelques épiceries de Moundou proposent des produits laitiers importés de type européen : de la poudre de lait, du lait concentré, du beurre pasteurisé et des fromages divers.

Le lait caillé (rouaba, en arabe local) est un lait fermenté écrémé. Il résulte du barattage total ou partiel du lait entier après fermentation. Lorsque le lait n'est pas baratté, le lait caillé est commercialisé après séparation de la crème présente à la surface (écrémage). Sur les marchés, la vente du lait caillé s'accompagne donc très fréquemment de la vente du beurre solide ou fondu (figure 4). Le beurre constitue un complément de revenu non négligeable pour les vendeuses de lait caillé.

Le lait caillé est très différent du lait entier fermenté (rayib, en arabe, ou, par abus de langage, yaourt local), commercialisé par les bars laitiers. Les femmes Peules ne semblent pas commercialiser le lait entier fermenté. Le tableau I présente la nomenclature des différents produits laitiers dans les trois langues parlées par les femmes Peules lors des enquêtes, l'arabe tchadien étant, de loin, la langue la plus utilisée sur les marchés.

D'après des sondages effectués le 2 juin 1999, le nombre de vendeuses de lait pénétrant dans la ville en une journée a été évalué à 373. Ces femmes portaient chacune en moyenne 12 litres de lait frais et de lait caillé répartis en 526 récipients dont 60 p. 100 contenaient le lait frais et le reste le lait caillé. Le marché de Moundou à cette saison peut donc être évalué à près de 5000 litres par jour. Des enquêtes complémentaires sont néanmoins nécessaires pour affiner cette estimation, étant donné les importantes variations saisonnières.

Le lait frais, le lait caillé et le beurre sont vendus sur les marchés de la ville ou commercialisés directement auprès des ménages. Le lait frais fait l'objet d'un commerce particulier auprès des quelques unités de transformation urbaines.

\section{Secteur de la transformation urbaine}

Il y a plusieurs petits ateliers de transformation à Moundou. Ce sont les bars laitiers (plus de 20 ont été recensés) qui vendent du lait entier fermenté, ainsi que deux fromageries artisanales.

Les bars laitiers fabriquent leur lait entier fermenté à partir de lait frais local. La fermentation se fait assez simplement dans des seaux, sans contrôle du ferment. Il est servi dans des chopes en verre, accompagné de sucre. Outre le lait entier fermenté, ces bars proposent des jus de fruits et des boissons gazeuses industrielles. Tous les cinq fabricants de lait entier fermenté rencontrés lors de l'étude trouvent leur activité rémunératrice. Avec une moyenne de 47 litres vendus par jour, ces bars écoulent environ 25 p. 100 de la production laitière locale commercialisée à Moundou.

Deux mini-fromageries fabriquent du fromage à partir de lait de collecte. Leurs procédés de fabrication diffèrent radicalement et correspondent, pour chacune d'elles, à un savoir-faire spécifique.

L'une des deux fromageries est l'atelier de Halou Hissen, situé au centre-ville. Il fabrique un fromage à pâte molle torsadé du type de celui connu à N'Djamena sous le nom de Pont-Bélilé. H. Hissen est venu s'installer en tant que fromager à Moundou en 1984. Il est le neveu de Rahmat Ab El Bagui, fondateur de l'entreprise familiale Zina de Pont-Bélilé. Cette entreprise avait hérité d'une famille de commerçants syriens le secret de fabrication d'un fromage syrien connu sous le nom de Gibna matfoula. H. Hissen mâ̂trise parfaitement cette technique de fabrication qui utilise notamment de la présure importée, présentée dans des tubes de comprimés (Samia rennet tablet, for cheese making, Red Label, Samman \& Kotoub, Damas, Syrie). Les quantités produites n'ont malheureusement pas pu être évaluées.

Le second type de fromage local vendu à Moundou est fabriqué par la communauté des sœurs de Donia, à $50 \mathrm{~km}$ de Moundou. Ce fromage à pâte molle se présente sous la forme d'une tome de $750 \mathrm{~g}$ environ. Là encore, il a été impossible d'évaluer les quantités produites. De plus, les auteurs n'ont pas été autorisés à visiter la fromagerie ni à discuter le procédé de fabrication de ce fromage (figure 5).

Pour comprendre le niveau de rentabilité de ces différentes entreprises de transformation, il convient d'étudier leur activité du point de vue comptable. A cette fin, les prix des différents produits sur les marchés de Moundou sont examinés ci-après.

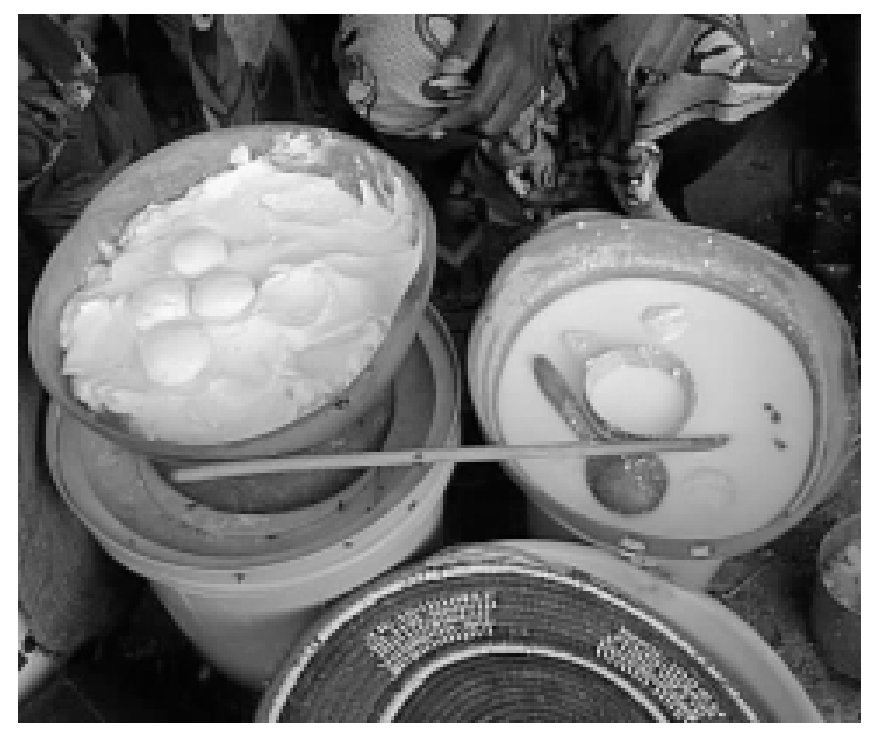

Figure 4 : vente du lait caillé et du beurre. 
Tableau I

N omenclature des produits laitiers traditionnels commercialisés à M oundou (source : enquêtes)

\begin{tabular}{llll} 
Français & Arabe tchadien & Foulfouldé & Haoussa \\
\hline Lait (nom générique) & Laban & Kossam & Madaro \\
Lait frais & Halib & Biradham & Nialloundé \\
Lait fermenté entier & Rayib & Nono \\
(yaourt local) & (ou Birquib en arabe littéraire) & Pendidam (Mastam ?) & Dian Nono \\
Lait fermenté écrémé & Rouaba & Lebol & Kindirmo \\
Beurre fermier solide & Zibda & Nihin baguar & Maï \\
Beurre fermier fondu & (littéralement, huile de vache) & $\begin{array}{l}\text { N ebam naii } \\
\text { (littéralement, huile de vache) }\end{array}$ & ou kaïnalam
\end{tabular}

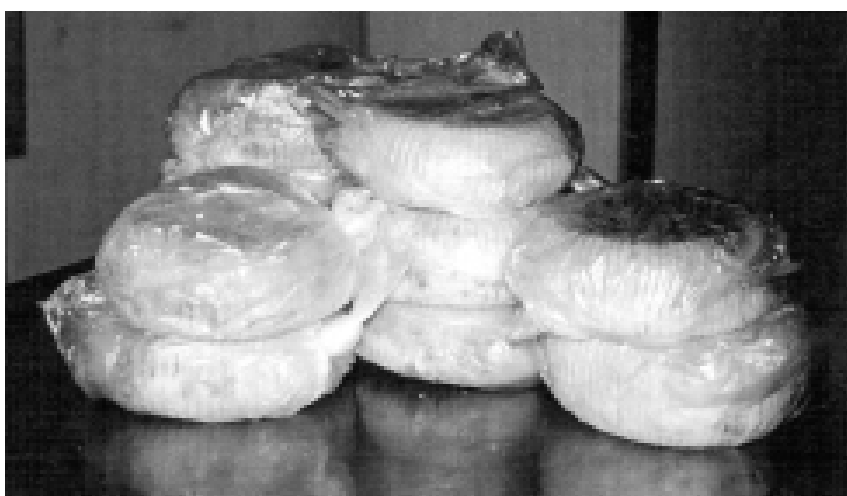

Figure 5 : fromage de Donia, un secret bien gardé.

\section{Comptabilité de la filière}

Le prix d'achat du lait frais est lié à la distance entre le lieu de vente et la ville. Sur les marchés situés en dehors de la zone actuelle de collecte il est très inférieur au prix de Moundou (tableau II)

En raison de l'enclavement du marché, la poudre de lait et les autres produits laitiers importés sont vendus à des prix élevés. La dévaluation du franc CFA en 1994 a d'ailleurs eu pour effet d'augmenter les prix relatifs de ces produits importés. Les coûts du transport varient avec l'état des routes et sont donc supérieurs en saison des pluies, période pendant laquelle le lait local est pourtant le moins cher. Le rapport des prix est donc globalement très favorable à la production locale, surtout en saison des pluies. Le tableau III indique les différentiels de prix constatés après enquêtes sur les marchés.

Les produits de luxe tels que le beurre de table ou les fromages sont aussi vendus à des prix élevés. Ce segment de marché est relativement étroit mais fortement rémunérateur. La cherté des importations permet aux transformateurs locaux de vendre leurs produits à des prix intéressants.

Le lait entier fermenté est vendu 600 FCFA le litre pour un prix d'achat du lait local variant entre 227 (saison des pluies) et 254 FCFA (saison sèche). H. Hissen commercialise son fromage au prix de 3250 FCFA par kilogramme à une épicerie qui le revend au détail à $3500 \mathrm{FCFA} / \mathrm{kg}$, pour un prix de revient en

\section{Tableau II}

Prix moyens du lait (en francs CFA par litre) sur différents marchés (d'après les personnes interrogées lors des enquêtes)

\begin{tabular}{lcccc} 
& Moundou & Donia & Bébalem & Laï \\
\hline Saison sèche & 233 & 167 & 167 & 133 \\
Saison des pluies & 200 & 133 & 133 & 100
\end{tabular}

\section{Tableau III}

Prix moyens du lait local et de la poudre sur le marché de Moundou (source : enquêtes)

\begin{tabular}{lcc} 
& $\begin{array}{c}\text { Saison des pluies } \\
\text { (FCFA/litre) }\end{array}$ & $\begin{array}{c}\text { Saison sèche } \\
\text { (FCFA/litre) }\end{array}$ \\
\hline $\begin{array}{l}\text { Lait frais local } \\
\begin{array}{l}\text { Poudre de lait N ido } \\
\text { (en équivalent lait) }\end{array}\end{array}$ & 200 & 233 \\
$\begin{array}{l}\text { Rapport de prix : } \\
\text { lait importé / lait local }\end{array}$ & 453 & 443 \\
& 2,2 & 1,9
\end{tabular}

matière première d'environ $1700 \mathrm{FCFA} / \mathrm{kg}$. Le fromage de Donia est vendu $4000 \mathrm{FCFA} / \mathrm{kg}$ par la mission catholique de Moundou. L'analyse rapide des comptabilités des transformateurs laitiers montre que cette activité est très rémunératrice.

\section{Consommation des produits laitiers}

Les enquêtes auprès des consommateurs montrent que les produits vendus par les Peuls sont utilisés selon des recettes traditionnelles : le lait caillé est incorporé dans la bouillie, le beurre est utilisé en cosmétique, notamment dans la chevelure, comme fortifiant pour les enfants ou bien dans la confection de sauces. Toujours selon les enquêtes, il semble que la réputation des femmes Fulata haoussa chez les consommateurs moundoulais soit bien meilleure que celle des autres femmes Peules du point de vue de l'hygiène des produits. 
Des enquêtes sur un échantillon plus large de consommateurs auraient été nécessaires pour évaluer quantitativement les effets des caractéristiques socioprofessionnelles sur la consommation de produits laitiers. Toutefois, les résultats de cette étude permettent de désigner les populations originaires du Nord comme celles qui consomment le plus de produits laitiers. Ceci s'explique bien sûr par le fait que l'élevage laitier est pratiqué surtout par des éleveurs originaires du nord du pays. Mais ces habitudes se diffusent, notamment chez les jeunes, sous l'influence du mode de vie urbain et des bars laitiers.

Les deux fromages de confection locale sont très appréciés des expatriés de Moundou, mais aussi de quelques ménages urbains à hauts revenus. Ce marché repose donc aussi sur des habitudes de consommation liées au mode de vie urbain.

\section{DISCUSSION}

La figure 6 synthétise les résultats des enquêtes sur la filière d'approvisionnement en produits laitiers de Moundou.

\section{Systèmes pastoraux en mutation}

Des évolutions lentes, tant du point de vue des techniques d'élevage que du point de vue des techniques laitières, montrent que les élevages étudiés sont en mutation. Le recours au tourteau témoigne d'une intensification de l'élevage, liée à l'augmentation de la pression de pâturages et aux perspectives du marché laitier.

Cette intensification butte néanmoins sur des difficultés d'approvisionnement en intrants, notamment en aliments concentrés. En effet, le marché du tourteau n'est pas concurrentiel : la société CotonTchad alloue annuellement des quantités de tourteaux à différents commerçants. Ces commerçants revendent le tourteau (après plusieurs intermédiaires) aux utilisateurs : éleveurs ou agro-industriels nigérians. Alors que le prix de vente sortie usine était au moment de l'enquête d'environ 850 FCFA par sac, le prix payé par les éleveurs est de plus de 1500 FCFA par sac (parfois plus de 2000 FCFA par sac). Devant cette situation, l'Association pour le développement de l'élevage au Tchad (Adet), le ministère de l'Elevage et d'autres institutions ont demandé qu'un quota de sacs soit alloué aux éleveurs chaque année par un comité ad hoc. Malgré cet effort, le jeu politique semble être en faveur des commerçants puisque l'Adet n'a réussi à obtenir que 30000 sacs en 1998. Toutefois, même au prix de 24,5 FCFA/kg, l'achat du tourteau semble intéresser les éleveurs.

Les techniques de transformation laitière Peules semblent elles aussi en évolution. Certains groupes d'éleveurs utilisent des techniques beaucoup plus élaborées que d'autres pour la fabrication

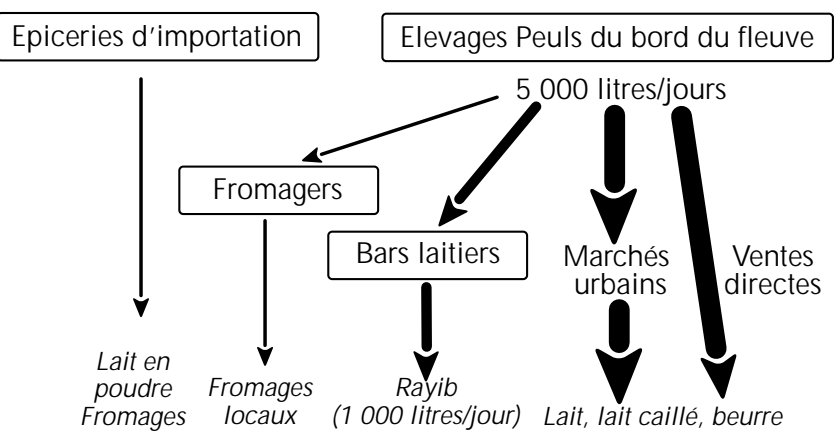

Consommateurs urbains

Figure 6 : schéma de la filière. des produits. C'est le cas du chauffage du lait après la traite qui permet d'obtenir un lait qui se conserve plus facilement. C'est aussi le cas de la technique de barattage de la crème qui représente une innovation intéressante. En effet, le barattage du lait fermenté sans séparation de la crème demande un travail assez long et pénible qui se fait en position assise : le barattage de six litres de lait prend environ 35 minutes, pour un rendement beurrier de seulement 60 p. 100 (12).

\section{Marché en expansion : rôle des petites et moyennes entreprises laitières}

L'analyse du marché montre que les perspectives de commercialisation du lait sont intéressantes. Les produits laitiers traditionnels sont vendus par les producteurs à des prix relativement élevés en comparaison du prix des produits laitiers dans d'autres pays du monde. D'autre part, le marché des produits transformés locaux (lait entier fermenté, fromages) est en pleine croissance.

Les petites entreprises de transformation laitières sont les principaux acteurs du développement du marché laitier : ce sont elles qui créent les nouveaux débouchés pour le lait de collecte. Il existe d'ailleurs de grandes potentialités pour collecter du lait en dehors du rayon actuel. Le développement des bars laitiers pourrait entraîner le développement de collecteurs à vélo ou à mobylette, comme cela a été le cas à N'Djamena.

Plusieurs facteurs freinent la multiplication des petites et moyennes entreprises laitières. En premier lieu, l'activité des fromagers repose essentiellement sur leur savoir-faire. Le non-accès à ce savoir-faire est une entrave au développement de cette activité. En second lieu, le développement des marchés du lait entier fermenté et des autres produits transformés de type européen est lié à l'évolution des caractéristiques de la demande. La diversification des habitudes alimentaires est un processus lent qui dépend de multiples facteurs parmi lesquels le niveau de vie des populations et l'évolution des modèles sociaux. Du statut de ville secondaire, Moundou pourrait rapidement évoluer vers celui de capitale régionale, ce qui se traduirait probablement par la multiplication des bars laitiers.

\section{Conflits sur l'utilisation de l'espace}

Les conflits entre éleveurs et agriculteurs sont évoqués de manière plus ou moins préoccupante selon les éleveurs interrogés. Ils peuvent être analysés aussi bien en termes de «gestion des ressources » que «d'opposition politique Nord-Sud» (1). Ces conflits posent le problème de l'intégration des communautés d'éleveurs originaires $\mathrm{du}$ Nord au contexte agricole autochtone, ce dernier étant d'ailleurs constitué de systèmes agraires dans lesquels l'élevage est très présent. Des enquêtes complémentaires semblent nécessaires pour mieux évaluer l'importance, les causes et les modes de gestion de ces conflits. Pour être pertinentes, ces enquêtes devront à la fois être menées auprès des éleveurs, des agriculteurs et des autorités qui interviennent dans l'attribution des terres et la gestion des conflits.

\section{CONCLUSION}

L'étude de la filière d'approvisionnement en produits laitiers de la ville de Moundou témoigne d'une intégration marchande plutôt réussie des éleveurs pastoraux. Cette intégration est originale dans la mesure où elle repose sur la vente du lait liquide, alors que les systèmes pastoraux sont plus souvent orientés vers la vente du 
bétail ou de services (transport). Le commerce laitier se fait ici à la faveur de l'installation des éleveurs transhumants en zone périurbaine et grâce à une intensification des techniques d'élevage. Une organisation plus efficace du marché du tourteau de coton pourrait même favoriser davantage cette intensification.

Toutefois, malgré ces changements, les élevages Peuls des environs de Moundou restent fondamentalement marqués par leur caractère pastoral : mobilité des troupeaux, difficulté d'accès au foncier, faiblesse du niveau de scolarisation, logement sous tentes traditionnelles. Ainsi, l'attraction du marché et l'influence urbaine impliquent une adaptation des systèmes sans remettre en cause leur mode de vie traditionnel.

La valorisation du lait local se fait soit à travers la commercialisation directe des produits traditionnels par les femmes d'éleveurs (sous-filière fermière), soit à travers la transformation du lait de collecte par les fromageries ou les bars laitiers (sous-filière artisanale). Dans ce contexte, les systèmes pastoraux prouvent qu'ils sont en mesure de répondre à la croissance rapide de la demande urbaine.

\section{BIBLIO GRAPHIE}

1. ARDITI C., 1999. Paysans Sara et éleveurs Arabes dans le sud du Tchad : du conflit à la cohabitation ? In : Barouin C., Boutrais J. Ed., L'homme et l'animal dans le bassin du lac Tchad. Paris, France, Ird Editions, p. 555-574.

2. ATTEYEH SOUGAL A., 1999. Le rôle des systèmes pastoraux dans l'approvisionnement en produits laitiers des marchés de deux villes de la zone soudanienne du Tchad (Moundou et Sahr). Mém. Dess, Université de Paris XII, France, 47 p. + annexes

3. CENTRES J.M., 1995. Stratégies de développement de la production laitière en Afrique. Tome 1 : Synthèse des études. Paris, France, Gret/ ministère de la Coopération, $122 \mathrm{p}$

4. COPPOCK D.L., HOLDEN S.J., MULU GETA ASSEFA, 1992. Review of dairy marketing and processing in a semi-arid pastoral system in Ethiopia. In: Brokken R., Senait Seyoum, Eds. Dairy marketing in Subsaharan Africa. Proc. Symp., ILCA, Addis-Abeba, Ethiopia, 26-30 november 1990. Addis-Abeba, Ethiopia, ILCA, p. 315-334.

5. DUTEURTRE G., 1998. Compétitivité prix et hors-prix sur le marché des produits laitiers d'Addis-Abeba (Ethiopie). Thèse d'Agro-économie, Ecole nationale supérieure agronomique, Montpellier, France, 361 p.

6. FAYE B., BENGOUMI M., HIDANE K., 1998. Le développement de l'élevage camelin laitier périurbain : I'exemple de Laâyoune (provinces sahariennes du Maroc). In : Atelier Marchés urbains et développement laitier, Cirad, M ontpellier, France, septembre 1998.

7. LE MASSO N A., 1992. Données techniques et socio-économiques sur les fromageries artisanales en République centrafricaine. Cah. Agric., $1: 270-277$
L'aide aux petites entreprises laitières pourrait contribuer à dynamiser la filière. L'augmentation de la demande en lait de collecte pourrait susciter un développement de nouveaux modes de transport du lait (à vélo ou à mobylette) qui permettraient d'élargir le rayon de collecte. La commercialisation du lait par les agriculteurs sédentaires possédant un petit cheptel pourrait alors être envisagée.

Toutefois la présence de l'élevage pastoral dans la région pose un certain nombre de problèmes liés à l'occupation de l'espace. Des actions pourraient être menées pour encourager la concertation entre éleveurs et agriculteurs sur l'utilisation des ressources. Audelà de l'intégration marchande, la question de l'intégration sociale et politique des élevages pastoraux reste posée.

\section{Remerciements}

Ces recherches ont été réalisées au Laboratoire de recherches vétérinaires et zootechniques (Lrvz) de Farcha dans le cadre du Pôle régional de recherches appliquées au développement des savanes d'Afrique centrale (Prasac).
8. METZER R., CENTRES J.M., THOMAS L., LAMBERT J.C., 1995. L'approvisionnement des villes africaines en lait et produits laitiers. Rome, Italie, Fao, 102 p. (Production et santé animale, $n^{\circ} 124$ )

9. MONTIGAUD J.C., 1989. Eléments de méthodologie des filières In : Griffon M. Ed., Economie des filières en régions chaudes. Formation des prix et échanges agricoles. Montpellier, France, Cirad-mesru, p. 38-49.

10. NELL A.J., 1992. An overview of dairying in Subsaharan Africa. In Brokken R., Senait Seyoum Eds., Dairy marketing in Subsaharan Africa. Proc. Symp. ILCA, Addis-Abeba, Ethiopia, 26-30 november 1990. Addis-Abeba, Ethiopia, ILCA, p. 31-44.

11. NJOYA A., LOKO DICKA B., 1997. Production laitière périurbaine des bovins au Nord Cameroun. In : Seiny Boukar L., Poulain J.F., Faure G. Eds. Agricultures des savanes du Nord Cameroun. Actes Atelier Garoua, Cameroun, 25-29 novembre 1996, p. 515-516.

12. ROY L., GAUTHIER I., ABO UBAKAR M., LE MASSO N A., 1998. La fabrication traditionnelle de beurre au Tchad. In : Atelier Marchés urbains et développement laitiers en Afrique subsaharienne, 9-10 septembre 1998. M ontpellier, France, Cirad, $6 p$.

13. VATIN F., 1996. Le lait et la raison marchande, essai de sociologie économique. Rennes, France, Presses universitaires, $205 \mathrm{p}$.

14. WALSHE M.J., GRINDLE J. NELL A., BACHMANN M. 1991. Dairy development in Subsaharan Africa: A study of issues and options. Washington, DC, USA, World Bank, 94 p. (Technical paper No. 135)

Reçu le 11.04.2000, accepté le 01.02.200 


\section{Summary}

Duteurtre G., Atteyeh A. Market integration of pastoral systems in the south of Chad: Case of the milk trade in Moundu

Since 1980, livestock husbandry has steadily increased in the south of Chad, particularly because of transhumant herd moves from the center to the south of the country. Supplying cities and villages with dairy products is an important trade for the breeders. This trade was the foundation for pastoral breeders to settle down around the city of Moundu. Can such a livestock system respond in a sustainable way to the quickly growing demand of the population? Does it change as a result of integrating the market? Suburban dairy producers, dairy processors, salespersons and consumers were surveyed to better understand the organization of this subsector. Results show that the traditional dairy product trade is dynamic. More than 5000 liters originating from suburban Fulani settlements reach city markets every day. The livestock system is also adapting itself well to the new trade environment, while keeping its intrinsic mobility, even on short distances. In addition, urban dairy processors (cheesemakers and dairy bars) boost this subsector. Finally, there is not much competition with imports due to the area being enclosed and consumers' preference for traditional products. However, Fulani pastoralist settlements generate some conflicts on land use. This study shows the need to initiate more talks between livestock breeders and cotton producers, to reorganize the cotton oilseed cake market, and to develop small dairy-processing units.

Key words: Milk product - Pastoralism - Marketing - Market Moundu - Chad.

\section{Resumen}

Duteurtre G., Atteyeh A. La leche en Moundu, testigo de la integración mercante de los sistemas pastoriles en el sur de Chad

Desde 1980, la importancia de la cría en la zona sudanesa de Chad no ha cesado de crecer debido, principalmente, al descenso de los hatos del centro hacia el sur del país. El abastecimiento de productos lecheros a las ciudades y pueblos de la zona, representa una actividad importante para estos establecimientos. Es sobre esta base que se establecieron poblaciones de criadores pastoriles alrededor de la ciudad de Moundu. ¿Pueden estos establecimientos responder de manera duradera al aumento rápido de la demanda urbana? ¿Serán estos sistemas de crianza modificados bajo el efecto de la integración al mercado? Encuestas realizadas con productores lecheros peri urbanos, transformadores de leche, comerciantes y consumidores, permiten una mejor comprensión de la organización de la filial. Los resultados muestran el dinamismo del comercio de los productos lecheros tradicionales y la importancia de los flujos de productos entre los campamentos peri urbanos Peuls y los mercados de la ciudad (más de 5000 litros por día). Estos sistemas de cría mostraron capacidades de adaptación al nuevo contexto mercantil, permaneciendo siempre ligados a su movilidad, incluso sobre distancias cortas. Por otro lado, la filial parece fuertemente dinamizada por el sector de la transformación lechera urbana (productores de queso y bares lecheros). Finalmente, la competencia de las importaciones es bastante baja, debido al enclave de la zona y a la preferencia de los consumidores por los productos tradicionales. Esta presencia de cría pastoril no está, sin embargo, exenta de problemas, como el que concierne la ocupación del espacio. Los resultados de este estudio subrayan las necesidades de reforzar las iniciativas de concertación entre criadores y agricultores, de favorecer una organización más eficaz del mercado de la torta de algodón, así como del desarrollo de los pequeños talleres de transformación.

Palabras clave: Leche - Producto lacteo - Pastoralismo Mercadeo - M ercado - Moundu - Chad. 